\title{
A Reason for the Earth Moving Away from the Sun and the Possibility to Escape Being Engulfed by a Red Giant Sun
}

\author{
Cui-Xiang Zhong \\ Department of Science and Technology, Jiangxi Normal University, Nanchang, China
}

\author{
Email address: \\ cuixiang_zhong@163.com
}

\section{To cite this article:}

Cui-Xiang Zhong. A Reason for the Earth Moving Away from the Sun and the Possibility to Escape Being Engulfed by a Red Giant Sun. American Journal of Astronomy and Astrophysics. Vol. 5, No. 3, 2017, pp. 25-27. doi: 10.11648/j.ajaa.20170503.11

Received: July 14, 2017; Accepted: July 24, 2017; Published: August 15, 2017

\begin{abstract}
Using the radar technology and the tracking technology of interplanetary spacecraft, scientists have calculated that the sun and Earth are gradually moving apart. But no one knows the reason why the Earth is moving away from the sun. So the author has analyzed various factors affecting Earth's movement, and found that during the normal rotation of the Earth around the sun, the atmospheric pressure on the trailing hemisphere of the Earth is higher than the other hemisphere, effectively increasing its rotation speed, thus making the Earth gradually move away from the sun along a spiral line. Generally, any planet with thick atmosphere can move away from its parent star under the sunshine of its parent star. At the current speed of Earth's moving away from the sun, the Earth can survive the sun's red giant phase.
\end{abstract}

Keywords: Sun, Earth, Red Giant, Orbit-Variation, Atmospheric Pressure

\section{Introduction}

As early as in the third century B C, some astronomers had found that the sun and Earth were gradually moving apart. With the progress of science and technology, scientists have further measured that the distance between the sun and Earth increases $15 \mathrm{~cm}$ per year. But as to the reason why the Earth gradually moves away from the sun, the scientific community has been arguing for a long time. Although many hypotheses have been proposed to explain the reason, none has proved satisfactory [1]. Hence, the author has researched deeply into various factors affecting Earth's movement, and has found that during the normal rotation of the Earth around the Sun, the atmospheric pressure on the trailing hemisphere of the Earth is higher than the other hemisphere, effectively increasing its speed, thus making the Earth gradually move away from the sun along a spiral-line. Moreover, at the current speed of Earth's moving away from the sun, the Earth can survive the sun's red giant phase.

\section{A Reason for the Earth Moving Away from the Sun}

In order to find the real reason for the Earth to move away from the sun, we should look for some heuristic information. In fact, after planetary scientists announced that they have observed a slight increase in the Earth's distance from the Sun, some people have made more careful observations [2]. "The Earth is being nudged out into a bigger orbit," said Dr. Biggy Lips. "Starting this summer, we noticed a small but detectable increase in the Earth's orbit. It's as if each day something is giving the Earth a little kick. The impulse is causing the Earth to speed up just so slightly, pushing the Earth to higher orbit. The jolt of energy is very small but builds up over time. To increase the orbit, the "push" would have to be on the trailing side of the Earth, effectively increasing its speed. This means the pulse of energy is occurring just before sunset." After days of careful seismic monitoring scientist believe the source of energy seems to be coming from the vicinity of Manhattan NY. "Some bouncing motion is occurring every afternoon," said Dr. Lips. "If we don't find the cause soon, and stop it, the Earth will be propelled completely out of the Solar System."

See how carefully these experts made their observations of the movement of the Earth. But one cloud is enough to eclipse all the sun, so they did not know the real cause of earth's orbit variation. Standers-by see more than gamesters. The author has analyzed various factors that cause Earth's orbit-variation, and has found that during the normal rotation of the Earth around the sun, the atmospheric pressure on the trailing 
hemisphere of the Earth is higher than the other hemisphere, as is shown in Fig. 1 (b), effectively increasing its speed, thus making the Earth gradually move away from the sun along a spiral line. This can be proved as follows.

During the earth's revolution around the sun and self-rotation, we can consider a hemisphere including 12 time-zones and using A to denote its central district, and call it "hemisphere A" and the other hemisphere "hemisphere B". When hemisphere A is in the daytime, as is shown by orange area in Fig. 1 (a), it's exposed to the sunlight, so the temperature of this hemisphere was generally higher than that of hemisphere B, making more vapor and other gas molecules evaporated from this hemisphere (especially such as a hemisphere centered around Manhattan that features a humid subtropical climate, near the source region for maritime tropical air) than from hemisphere B, many of

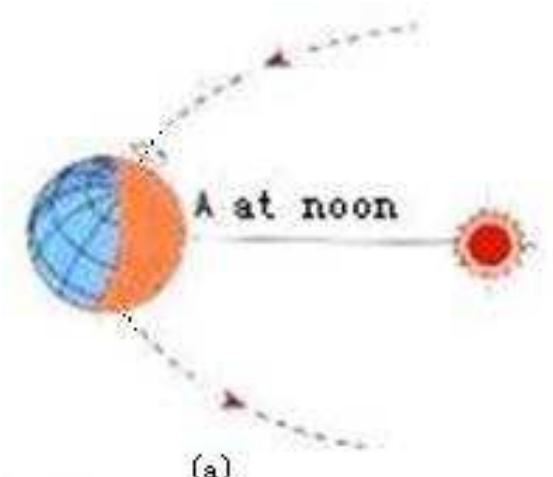

(a) these molecules may rise high into the sky and cool and condense into clouds, which then move with the rotation of the Earth. While hemisphere B undergoing the cooling of night, many gas molecules condense into dew and fall to the ground, causing the temperature of this hemisphere to drop a lot. Hence, when district $\mathrm{A}$ is at sunset, as is shown in Fig. 1 (b), the temperature of hemisphere $\mathrm{A}$ is generally higher than that of hemisphere B, so it's much easier for water vapor above hemisphere $\mathrm{B}$ to condense into water droplets than that above hemisphere A. Hence, the atmospheric pressure of hemisphere $\mathrm{A}$ is higher than that of hemisphere $\mathrm{B}$, which is equivalent to exerting a thrust to the trailing side of Earth along the direction of the Earth's revolution, effectively increasing Earth's speed, making the Earth gradually move away from the sun along a spiral line.

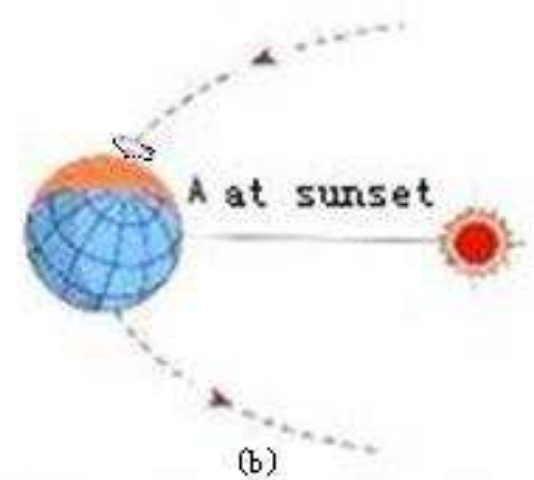

(b)

Figure 1. (a) When hemisphere $A$ is in the daytime (b) When district $A$ is at sunset.

\section{The Possibility for the Earth to Escape Being Engulfed by a Red Giant Sun}

In about 5 to 6 billion years, the Sun will have depleted the hydrogen fuel in its core and will begin to expand. At its largest, its surface will approximately reach the current orbit of Earth. The evolution of the sun into and through the red-giant phase has been extensively modeled, but it remains unclear whether Earth will be engulfed by the sun or will continue in orbit $[3,4,5,6,7]$.

According to the research result described in the previous section, we know that during the normal rotation of the Earth around the sun, the atmospheric pressure on the trailing hemisphere of the Earth is higher than the other hemisphere, effectively increasing its speed, thus making the Earth move away from the sun along a spiral line. At a speed of $15 \mathrm{~cm}$ per year, in about 5 to 6 billion years, the Earth will move 750,000 $\mathrm{km}$ away from the current orbit, so there is no danger of being engulfed by the sun as a red giant.

In addition, when the sun evolves to the end of the main sequence stage, its mass increase greatly. The greater a star's mass is, the faster its resource is consumed. But old star usually has complex hierarchical subsystems including many planets, satellites and even some stars, which move around the old star and grab a lot of resource originally belong to the old star. When the hydrogen needed for the thermonuclear reactions in the central region of the old star is insufficient, the thermonuclear reactions stop gradually and so the core begins to contract due to its own gravity. Due to the conservation of angular momentum, the reduction of the moment of inertia causes the old star's rotation speed increasing rapidly, making the Earth's revolution speed increase accordingly, thus making the Earth move away from the sun quickly. Hence, in view of this, there is no danger of being engulfed by the sun as a red giant $[8,9,10]$.

\section{Conclusions}

During the earth's revolution around the sun and self-rotation, the atmospheric pressure on the trailing hemisphere of the Earth is higher than the other hemisphere, effectively increasing its speed, making the Earth gradually move away from the sun along a spiral line. Thus, we can see the reason for Earth moving away from the sun is the change of atmospheric pressure of the earth under sunshine. Some other planets in the Solar system, such as Mars, Jupiter, Saturn, Uranus and Neptune, also have dense atmosphere, their atmospheric pressures will also change under sunshine, thus making them move away from the sun. At the current speed of Earth's moving away from the sun, the Earth can survive the sun's red giant phase.

In addition, when the sun evolves to the end of the main sequence stage, its core begins to contract and consequently its 
rotation speed increasing rapidly, making the Earth's revolution speed increase accordingly, thus also making the Earth move away from the sun quickly.

\section{References}

[1] Kelly Beatty. Why is the Earth moving away from the sun? [J]. New scientist, June 2009.

(https://www.newscientist.com/article/dn17228-why-is-the-ear th-moving-away-from- the-sun)

[2] Beau Gusphax. Earth Moving Away from the Sun [J]. Scientific Proof Magazine, December 23, 2010. (https://scientificproofmagazine.com/2010/12/23/earth-movin g-away-from-the-sun/)

[3] Zeilik, Michael A.; Gregory, Stephan A. Introductory Astronomy \& Astrophysics (4th ed.). Saunders College Publishing, 1998, pp. 321-322.

[4] Sackmann, I. -J.; Boothroyd, A. I.; Kraemer, K. E. Our Sun. III. Present and Future [J]. The Astrophysical Journal, 1993, 418: 457.
[5] Laughlin, G.; Bodenheimer, P.; Adams, F. C. The End of the Main Sequence [J]. The Astrophysical Journal, 1997, 482: 420.

[6] Ramirez, Ramses M.; Kaltenegger, Lisa. Habitable Zones of Post-Main Sequence Stars [J]. The Astrophysical Journal, 2016, $823(1)$

[7] Jones, M. I.; Jenkins, J. S.; Bluhm, P.; Rojo, P.; Melo, C. H. F. The properties of planets around giant stars [J]. Astronomy \& Astrophysics, 2014. 566: A 113.

[8] Cui-Xiang Zhong. The Formation and Evolution of Galaxies and the Expansion of the Universe as Well as Dark Matter \&Dark Energy [J]. AASCIT Journal of Physics, 2016, 2 (3): 18-26. Published online, Mar. 2, 2016 (http://www.aascit.org/journal/archive2?journalId=977\&paper $\mathrm{Id}=4307)$.

[9] Reiners, A.; Basri, G. On the magnetic topology of partially and fully convective stars. Astronomy and Astrophysics, 2009. 496 (3): 787.

[10] Lopez, Bruno; Schneider, Jean; Danchi, William C. Can Life Develop in the Expanded Habitable Zones around Red Giant Stars? The Astrophysical Journal, 2005. 627(2): 974-985. 